\section{RSP}

http://www.rsp.fsp.usp.br/
Revista de Saúde Pública

\title{
Poluição do ar e hospitalizações na maior metrópole brasileira
}

\author{
Nelson Gouveia', Flavia Prado Corrallo", Antônio Carlos Ponce de Leon"', Washington Junger"I, \\ Clarice Umbelino de Freitas ${ }^{\mathrm{IV}}$ \\ I Universidade de São Paulo. Faculdade de Medicina. Departamento de Medicina Preventiva. São Paulo, SP, Brasil \\ " Coordenadoria de Vigilância a Saúde do Município de Diadema. Núcleo de Vigilância em Saúde Ambiental. \\ Diadema, SP, Brasil \\ III Universidade Estadual do Rio de Janeiro. Instituto de Medicina Social. Rio de Janeiro, RJ, Brasil \\ iv Universidade de São Paulo. Faculdade de Medicina. Hospital das Clinicas. Laboratório de Investigação Médica - \\ LIM 39. São Paulo, SP, Brasil
}

\section{RESUMO}

OBJETIVO: Avaliar o impacto da poluição do ar na maior metrópole brasileira sobre as internações por doenças respiratórias e cardiovasculares.

MÉTODOS: Foi realizado estudo com dados das estações de monitoramento de nove municípios da Região Metropolitana de São Paulo, tendo o PM $_{10}$ como indicador de poluição e as internações por doenças respiratórias e cardiovasculares como indicadores de efeito. Para cada município foi realizada uma análise de séries temporais usando modelos explicativos para contagens de internações ao longo do tempo via regressão de Poisson semi-paramétrica. Os resultados foram combinados em uma meta-análise de modo a estimar o risco global do $\mathrm{PM}_{10}$ na Região Metropolitana de São Paulo.

RESULTADOS: Todos os municípios apresentaram estimativas estatisticamente significantes para as hospitalizações por doenças respiratórias com exceção de Santo André e Taboão da Serra. Os riscos relativos de admissão hospitalar para um aumento de $10 \mu \mathrm{g} / \mathrm{m}^{3}$ nos níveis de $\mathrm{PM}_{10}$ variaram de 1,011 (IC95\% 1,009-1,013) para São Paulo a 1,032 (IC95\% 1,024-1,040) em São Bernardo do Campo, para doenças respiratórias totais. O risco de internação por doenças respiratórias em crianças variou de 1,009 (IC95\% 1,001-1,017) em Santo André a 1,077 (IC95\% 1,056-1,098) em Mauá. Somente São Paulo e São Bernardo do Campo apresentaram resultados positivos e estatisticamente significantes para internações por doenças cardiovasculares.

CONCLUSÕES: Esse é o primeiro estudo a estimar o risco de adoecimento devido à poluição do ar no conjunto de municípios da Região Metropolitana de São Paulo. As estimativas globais do efeito da exposição à poluição na região indicaram associações somente com as doenças respiratórias. Apenas São Paulo e de São Bernardo do Campo mostraram associação entre os níveis de $\mathrm{PM}_{10}$ e as internações por doenças cardiovasculares.

DESCRITORES: Poluição do Ar, efeitos adversos. Doenças Respiratórias, epidemiologia. Doenças Cardiovasculares, epidemiologia. Metanálise. 


\section{INTRODUÇÃO}

A Região Metropolitana de São Paulo (RMSP), também conhecida como Grande São Paulo, reúne 39 municípios do estado de São Paulo, compreendendo uma área de 7.946 km², com uma população para o ano de 2010 de aproximadamente 20 milhões de habitantes (Figura 1)a. A região conta com uma frota veicular circulante estimada em 2012 de 6,9 milhões de veículos, o que corresponde a $49 \%$ da frota do estado e possui a maior concentração de indústrias do Brasil, fatores que a colocam no centro das atenções no que se relaciona à poluição atmosférica e ao estudo de seus impactos a partir de fontes móveis e fixas .

Essas fontes poluidoras foram responsáveis pelas emissões para a atmosfera de aproximadamente 132 mil t/ano de monóxido de carbono (CO), 42 mil t/ano de hidrocarbonetos (HC), 77 mil t/ano de óxidos de nitrogênio $\left(\mathrm{NO}_{\mathrm{x}}\right)$, 4,5 mil t/ano de material particulado (PM) e 11 mil t/ano de óxidos de enxofre ( $\mathrm{SO}_{\mathrm{x}}$ ), sendo os veículos responsáveis por $97 \%$ das emissões de $\mathrm{CO}, 81 \%$ de $\mathrm{HC}, 80 \%$ de $\mathrm{NO}_{\mathrm{x}}, 48 \%$ de $\mathrm{SO}_{\mathrm{x}}$ e $40 \%$ de $\mathrm{PM}^{\mathrm{c}}$. Vale ressaltar que a RMSP é responsável por aproximadamente $40 \%$ das emissões de monóxido de carbono (CO), hidrocarbonetos não

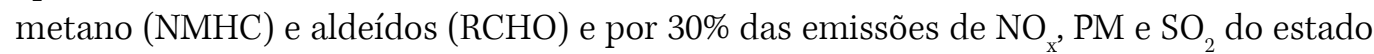
de São Pauloc.

Dessa forma, para acompanhar a evolução da qualidade do ar e orientar medidas de controle ambiental, a Companhia Ambiental do Estado de São Paulo (CETESB) instalou uma rede de monitoramento da qualidade do ar na RMSP, com 26 estações fixas, distribuídas em 10 municípios (Carapicuíba, Diadema, Guarulhos, Mauá, Osasco, Santo André, São Bernardo do Campo, São Caetano do Sul, São Paulo e Taboão da Serra). Em 23 dessas estações, o material particulado inalável $\left(\mathrm{PM}_{10}\right)$ é o poluente aferido .

a Fundação Sistema Estadual de Análise de Dados. Portal de Estatísticas do Estado de São Paulo: tabelas da Região Metropolitana do Estado de São Paulo. São Paulo: SEADE; 2010 [citado 1 jul 2016]. Disponível em: http://www.imp.seade.gov. br/frontend/\#/tabelas

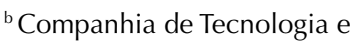
Saneamento Ambiental de São Paulo. Relatório da Qualidade do Ar 2013. São Paulo: CETESB; 2014 [citado 5 jul 2016].

Disponível em: http://www. cetesb.sp.gov.br/ar/qualidade-doar/31-publicacoes-e-relatorios

${ }^{\mathrm{c}}$ Companhia de Tecnologia e Saneamento Ambiental de São Paulo. Emissões veiculares no Estado de São Paulo 2013. São Paulo: CETESB; 2014 [citado 5 jul 2016]. Disponível em: http://veicular.cetesb. sp.gov.br/wp-content/uploads/ sites/35/2013/12/relatorioemissoes-veiculares-2013.pdf

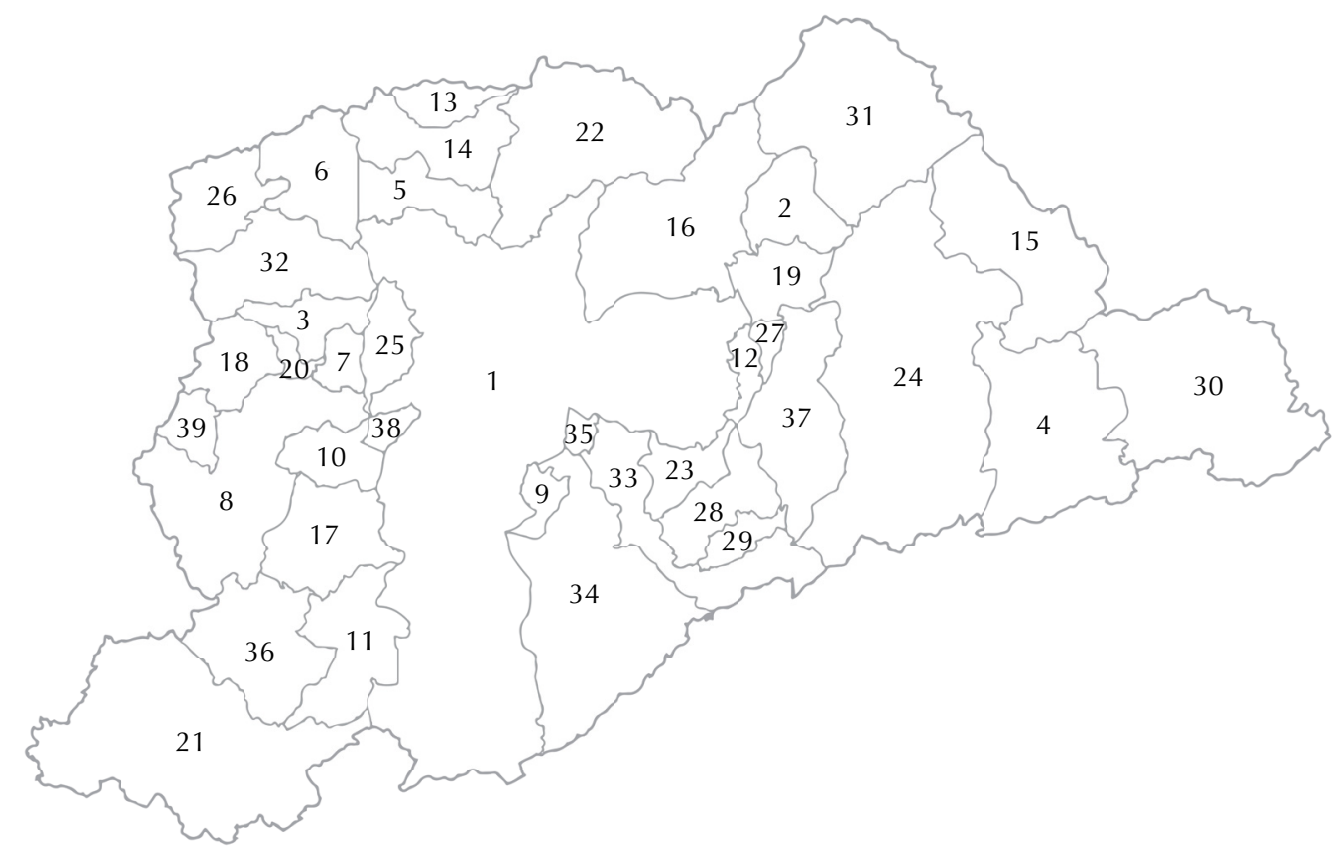

1 - São Paulo

2 - Arujá

3 - Barueri

4 - Biritiba-Mirim

5 - Caieiras

6 - Cajamar

7 - Carapicuiba

8 - Cotia

9 - Diadema

10 - Embu

11 - Embu-Guaçu
12 - Ferraz de Vasconcelos
13 - Francisco Morato
14 - Franco da Rocha
15 - Guararema
16 - Guarulhos
17 - Itapecerica da Serra
18 - Itapevi
19 - Itaquaquecetuba
20 - Jandira

21 - Juquitiba

22 - Mairiporã

23 - Mauá

24 - Mogi das Cruzes

25 - Osasco

26 - Pirapora do Bom Jesus

27 - Poá

28 - Ribeirão Pires

29 - Rio Grande da Serra

30 - Salesópolis
31 - Santa Isabel

32 - Santana de Parnaiba

33 - Santo André

34 - São Bernardo do Campo

35 - São Caetano do Sul

36 - São Lourenço da Serra

37 - Suzano

38 - Taboão da Serra

39 - Vargem Grande Paulista
Figura 1. Municípios que compõem a Região Metropolitana de São Paulo. 
Inúmeros estudos têm mostrado o impacto da exposição ao $\mathrm{PM}_{10}$ na saúde, com destaque para as doenças respiratórias e cardiovasculares. Dentre os desfechos respiratórios, a exposição ao $\mathrm{PM}_{10}$ associa-se ao incremento de internações por asma, pneumonia e doença pulmonar obstrutiva crônica ${ }^{10}$, assim como à exacerbação de sintomas associados a doenças alérgicas respiratórias ${ }^{17}$. Já em relação as doenças cardiovasculares (DCV), atribui-se à exposição ao $\mathrm{PM}_{10} \mathrm{o}$ aumento de internações hospitalares e óbitos por acidente vascular cerebral ${ }^{1,15} \mathrm{e}$ insuficiência cardíaca congestiva ${ }^{7}$, internações e atendimento em serviços de emergência por hipertensão $0^{11} \mathrm{e}$ arritmia ${ }^{20} \mathrm{e}$ hospitalizações por infarto agudo do miocárdio ${ }^{10}$.

Além dos efeitos à saúde acima descritos, foi encontrada correlação entre $\mathrm{PM}_{10} \mathrm{e}$ incidência de câncer (pele, pulmão, tireoide, laringe e bexiga) e óbitos por câncer de pulmão ${ }^{23}$, incidência de trombose venosa profunda ${ }^{2}$, partos prematuros ${ }^{12}$, baixo peso ao nascer ${ }^{9,14}$, aumento da mortalidade neonatal ${ }^{13}$ e como fator de exposição importante no desenvolvimento precoce da aterosclerose ${ }^{18}$ e desencadeamento de crises álgicas em portadores de anemia falciforme ${ }^{3}$.

Dentre os municípios da RMSP que contam com monitoramento da qualidade do ar, o município de São Paulo conta com diversas publicações sobre os efeitos deletérios da contaminação atmosférica. Mais recentemente, estudos no município de Santo André mostraram associação entre poluição e internações por insuficiencia cardíaca ${ }^{7,16}$, baixo peso ao nascer ${ }^{19} \mathrm{e}$ aumento da pressão arterial em controladores do tráfego ${ }^{5}$.

Dessa forma, no sentido de avaliar o impacto da poluição do ar no conjunto da RMSP, foi realizado estudo com dados dessas estações de monitoramento, tendo o $\mathrm{PM}_{10}$ como indicador de poluição e as internações por doenças respiratórias e cardiovasculares como indicadores de efeito. Os resultados foram então combinados em uma meta-análise, de modo a estimar o risco global do $\mathrm{PM}_{10}$ na região. Estimativas do impacto global da poluição do ar na população da RMSP são importantes no sentido de subsidiar discussões a respeito de intervenções que visem o controle dessa exposição.

\section{MÉTODOS}

Os dados utilizados para essas análises foram extraídos do portal de informações cuja construção foi financiada pelo Ministério da Saúde para a realização do Projeto de Avaliação de Impacto da Poluição do Ar nas Cidades Brasileiras (http://www.observandosaopaulo. com.br). Nesse portal, foram disponibilizados dados agrupados por dia das Autorizações de Internação Hospitalar (AIH) segundo local de residência, sistematizados pelo Departamento de Informática do Sistema Único de Saúde (DATASUS), no período de 2000 a 2008. Além disso, esse portal disponilibizou também dados dos poluentes atmosféricos coletados pela estações de monitoramento da CETESB, bem como os dados meteorológicos provenientes do Instituto de Astronomia e Geofísica (IAG) da Universidade de São Paulo e da CETESB. Foram utilizadas como variáveis dependentes as contagens diárias de internações por doenças respiratórias (CID10: J00-J99) em todas as idades (DRT), doenças respiratórias (CID10: J00-J99) em menores de cinco anos (DRC) e doenças cardiovasculares (CID10: I00-I99) em maiores de 39 anos (DCV). Alguns municípios apresentavam grande variabilidade no volume de internações, com média diária muito pequena e com muitos dias sem eventos, o que impossibilita a análise. Dessa forma, estabeleceu-se, como regra geral para inclusão do município no Projeto de Avaliação de Impacto da Poluição do Ar nas Cidades Brasileiras, que:

- municípios com média diária de três ou mais eventos/dia seriam analisados;

- para municípios com média diária menor de que três eventos/dia mas maior do que um, levou-se em conta o ajuste do modelo. Não havendo ajuste, o resultado seria analisado com ressalvas; e

- municípios com média diária de eventos menor que um não seriam analisados. 
Na RMSP, nem todos os municípios monitoram o conjunto de poluentes regulamentados. Optou-se então por analisar o $\mathrm{PM}_{10}$ como variável de exposição nos diversos municípios, de forma a permitir a sumarização da estimativa de risco, relativa a esse poluente para a região. As medidas de $\mathrm{PM}_{10}$ cedidas, originalmente de forma horária, foram agregadas ao banco de dados em médias diárias. O município de São Paulo conta com 13 estações de monitoramento da qualidade do ar que realizam medições de $\mathrm{PM}_{10}$ e os municípios de Santo André e Guarulhos, com duas estações cada. Nos demais municípios, existe apenas uma estação de monitoramento da qualidade do ar da CETESB que mede o $\mathrm{PM}_{10}$.

Considerou-se, para fins de análise, a média do dia do conjunto das estações para aqueles municípios que apresentavam mais de uma estação de monitoramento da qualidade do ar. As bases de dados das estações de monitoramento da CETESB apresentam lacunas nos valores dos poluentes, tendo muitas vezes grandes períodos sem informação. Devido a isso, as bases foram avaliadas quanto à completude dos dados do $\mathrm{PM}_{10}$, tendo-se buscado estabelecer um período mínimo de análise de três anos consecutivos, aceitando-se perdas de informações do poluente de, no máximo, $15 \%$ dos dias. Tivemos como consequência períodos variáveis de análise dos municípios, em detrimento do inicialmente proposto, que era avaliar o impacto da poluição do ar na saúde da população da RMSP para o período de 2000 a 2008. Pela mesma razão, o município de Carapicuíba não foi incluído na presente análise, pois não apresentava dados disponíveis para o período 2000 a 2008.

A temperatura e a umidade relativa do ar foram utilizadas como controles nas análises, mas cabe ressaltar que a maior parte dos municípios da RMSP não conta com estações meteorológicas. As medidas de temperatura e umidade relativa do ar, utilizadas para os diversos municípios, foram as mesmas utilizadas para o município de São Paulo, tendo-se trabalhado com a média das médias, média das mínimas e média das máximas das estações da CETESB e do IAG. Os municípios de Guarulhos e São Caetano do Sul, mesmo contando com estações medidoras de temperatura e umidade, apresentaram grandes lacunas de informação, o que inviabilizou a utilização desses dados.

Foi realizada análise de séries temporais com a biblioteca ARES, desenvolvida para o aplicativo $\mathrm{R}^{\mathrm{d}}$, utilizando metodologia comum para as localidades que aderiram ao "Projeto de Avaliação de Impacto da Poluição do Ar nas Cidades Brasileiras”.

Foram construídos modelos explicativos para contagens de internações pelas causas pesquisadas ao longo do tempo para cada município participante do estudo. Os modelos propostos pertencem à classe de Modelos Aditivos Generalizados (GAM), com a opção de regressão de Poisson, segundo a equação:

$$
\ln \left(E\left(Y_{t}\right)\right)=\beta X_{1 t}+\sum_{i=2}^{p} S_{i}\left(X_{i t}\right)
$$

onde $Y_{t}$ e $X_{1 t}$ são os números de eventos mórbidos e o nível de um dado poluente no dia $t$, respectivamente; $X_{i t}$ são as variáveis preditoras, que inclui o tempo; e $S_{i}$ são as funções de suavização, utilizando natural splines. Foram acrescentadas também variáveis indicadoras (dicotômicas) para os dias da semana e feriados nacionais ou locais, tendo-se testado a sua significância. Os feriados com significância de até 0,09 foram agrupados segundo a direção do seu efeito: positiva ou negativa. No processo de modelagem da série temporal, buscou-se minimizar o Critério de Informação de Akaike (AIC) e otimizar a função de autocorrelação parcial (PACF).

dJunger WL. Análise, imputação de dados e interfaces computacionais em estudos de séries temporais epidemiológicas [tese]. Rio de Janeiro: Instituto de Medicina Social da Universidade Estadual do Rio de Janeiro; 2008 [citado 10 jul 2017]. Disponível em: http://www.bdtd.uerj.br/ tde_arquivos/44/TDE-2013-0516T162258Z-3184/Publico/ Washington\%20Leite\%20Jungertese.pdf
Após a construção do modelo de trabalho de cada município (Core Model), que continha todas as variáveis de controle e da verificação de sua adequação, foi introduzido o $\mathrm{PM}_{10} \mathrm{em}$ defasagens de até cinco dias (lag simples) e verificado também o efeito cumulativo nesse período, utilizando um modelo polinomial de defasagens distribuídas. Esse modelo, além de considerar a latência do efeito dos poluentes, minimiza a instabilidade no processo de estimação, próprio das análises que utilizam múltiplas defasagens ${ }^{21}$. 
As estimativas de risco foram calculadas a partir da introdução da variável explanatória no modelo de trabalho de forma linear. Esse modelo estatístico fornece o risco relativo percentual para cada incremento de $10 \mu \mathrm{g} / \mathrm{m}^{3}$ nos níveis de $\mathrm{PM}_{10}$. Em todas as análises, assumiu-se nível de significância de $5 \%$.

O passo seguinte foi realizar a meta-análise dos coeficientes encontrados, considerando efeitos aleatórios, método de DerSimonian e Laird ${ }^{6}$ com estimativa de heterogeneidade proposta por Mantel-Haenszel, uma vez que a hipótese de homogeneidade foi rejeitada em todos os casos. A meta-análise foi realizada no Stata 12, por meio da macro metan, que permite a realização de meta-análise de efeitos fixos ou aleatórios.

\section{RESULTADOS}

A Tabela 1 apresenta o número médio de internações por doenças respiratórias e cardiovasculares, assim como a análise descritiva dos níveis de $\mathrm{PM}_{10}$, para cada uma das nove cidades analisadas. Com exceção do município de São Paulo, os demais municípios apresentaram números relativamente pequenos de internações diárias, porém apenas São Caetano do Sul teve média inferior a 1 para internações por doenças respiratórias em crianças. Guarulhos e Osasco apresentaram os maiores valores médios de $\mathrm{PM}_{10}$, e o percentual de perdas para essa variável foi relativamente baixo para todos os municípios.

Uma vez que para cada município foi construído um modelo explicativo, houve grande variação no número de graus de liberdade utilizados para o controle da tendência e sazonalidade, o que esteve relacionado ao período de análise e aos menores AIC, em conjunto com a melhor otimização da PACF. Como exemplo, no município de São Paulo, com maior tempo de análise foram utilizados 36 graus de liberdade para as doenças respiratórias totais, enquanto para o município de Taboão da Serra foram utilizados apenas 12. O controle da temperatura e umidade foi obtido em cada município ora utilizando-se o valor do dia de cada um dos parâmetros, ora a média de dois dias ou o lag simples até dois dias. Para esses parâmetros, foram utilizados poucos graus de liberdade, chegando-se no máximo a cinco em Mauá.

As estimativas de risco relativo para os efeitos cumulativos de zero a cinco dias, utilizando-se o modelo polinomial de defasagens distribuídas, encontram-se na Tabela 2. Excetuando-se os municípios de Santo André (DRT) e Taboão da Serra (DRC), os demais municípios apresentaram estimativas estatisticamente significantes para as hospitalizações por doenças respiratórias. Os riscos relativos de admissão hospitalar para um aumento de $10 \mu \mathrm{g} / \mathrm{m}^{3}$ nos níveis de $\mathrm{PM}_{10}$ variaram de 1,011 (IC95\% 1,009-1,013) para São Paulo a 1,032 (IC95\% 1,024-1,040) em São Bernardo do Campo, para doenças respiratórias totais. O risco de internação por doenças respiratórias em crianças variou de 1,009 (IC95\%1,001-1,017) em Santo André a 1,077 (IC95\%1,056-1,098) em Mauá.

Tabela 1. Parâmetros básicos de análise: médias diárias de internações, período de estudo e análise descritiva do PM 10 nos municípios pesquisados.

\begin{tabular}{|c|c|c|c|c|c|c|c|c|c|c|}
\hline Município & $\begin{array}{l}\text { Doenças } \\
\text { respiratórias }\end{array}$ & $\begin{array}{c}\text { Doenças } \\
\text { cardiovasculares }\end{array}$ & $\begin{array}{l}\text { Respiratórias } \\
\text { em }<5 \text { anos }\end{array}$ & Período de análise & $\begin{array}{l}\text { Média } \\
\text { PM }_{10}\end{array}$ & DP & P25 & P50 & P75 & $\begin{array}{c}\% \\
\text { Perdas } \\
\text { PM }_{10}\end{array}$ \\
\hline Diadema & 7 & 5 & 3 & $1 / 2004$ a $12 / 2008$ & 31,9 & 21,4 & 17,0 & 27,0 & 41,0 & 4,16 \\
\hline Guarulhos & 15 & 12 & 6 & $1 / 2003$ a $12 / 2005$ & 59,5 & 26,2 & 41,7 & 54,2 & 71,7 & 8,67 \\
\hline Mauá & 5 & 4 & 2 & 1/2001 a 12/2007 & 36,6 & 19,0 & 23,1 & 33,2 & 47,1 & 4,85 \\
\hline Osasco & 7 & 7 & 3 & $1 / 2000$ a $12 / 2008$ & 58,0 & 29,0 & 37,7 & 51,8 & 73,1 & 4,93 \\
\hline São Bernardo do Campo & 8 & 9 & 3 & $1 / 2004$ a $12 / 2006$ & 36,6 & 20,6 & 22,7 & 30,9 & 44,3 & 1,92 \\
\hline São Caetano do Sul & 3 & 3 & 0,8 & $1 / 2000$ a $12 / 2006$ & 38,9 & 19,8 & 24,7 & 34,5 & 48,8 & 5,16 \\
\hline São Paulo & 135 & 122 & 61 & $1 / 2000$ a $12 / 2008$ & 43,9 & 20,6 & 29,0 & 38,9 & 54,6 & 0 \\
\hline Santo André & 6 & 7 & 2 & $1 / 2000$ a $12 / 2008$ & 34,3 & 20,3 & 19,9 & 27,0 & 43,3 & 0,67 \\
\hline Taboão da Serra & 3 & 2 & 1 & 1/2002 a 12/2004 & 40,8 & 24,1 & 23,7 & 54,2 & 52,3 & 5,84 \\
\hline
\end{tabular}

Fonte: DATASUS e CETESB. 
Por outro lado, as estimativas para internações por DCV foram estatisticamente significantes apenas para os municípios de São Paulo, São Bernardo do Campo e Santo André. Em Santo André, observou-se um risco relativo menor do que 1. As demais estimativas, além de não serem estatisticamente significantes, apresentaram grande variação (Tabela 2).

A meta-análise dos três desfechos estudados indicou heterogeneidade entre as estimativas individuais de cada município; sendo assim, modelos de efeitos aleatórios foram estimados para obtenção das estimativas sumarizadas. Os resultados da meta-análise estão apresentados nas Figuras 2, 3 e 4.

Observa-se que as estimativas globais de efeito do $\mathrm{PM}_{10}$ para um acréscimo de $10 \mu \mathrm{g} / \mathrm{m}^{3}$ foram estatisticamente significantes e de magnitude semelhante para as hospitalizações por doenças respiratórias em todas as idades $(\mathrm{RR}=1,014$, IC95\% 1,009-1,019) e para crianças menores de cinco anos $(\mathrm{RR}=1,019,1,012-1,025)$. Por outro lado, a estimativa resumo para as hospitalizações por DCV foi aproximadamente 1 e não estatisticamente significante.

Tabela 2. Riscos relativos (RR) e intervalo de confiança (IC95\%) para um aumento de $10 \mu \mathrm{g} / \mathrm{m}^{3}$ nos níveis de $\mathrm{PM}_{10}$ para cada desfecho nos municípios estudados.

\begin{tabular}{|c|c|c|c|c|c|c|}
\hline \multirow{2}{*}{ Município } & \multicolumn{2}{|c|}{ Doenças respiratórias totais } & \multicolumn{2}{|c|}{$\begin{array}{c}\text { Doenças respiratórias totais } \\
\qquad 5 \text { anos }\end{array}$} & \multicolumn{2}{|c|}{ Doenças cardiovasculares } \\
\hline & $\mathbf{R R}$ & IC95\% & $\mathbf{R R}$ & IC95\% & $\mathbf{R R}$ & IC95\% \\
\hline Diadema & 1,012 & $1,007-1,017$ & 1,012 & $1,005-1,020$ & 1,001 & $0,995-1,007$ \\
\hline Guarulhos & 1,017 & $1,012-1,021$ & 1,010 & $1,002-1,017$ & 0,996 & $0,992-1,001$ \\
\hline Mauá & 1,021 & $1,008-1,033$ & 1,077 & $1,056-1,098$ & 0,956 & $0,944-0,969$ \\
\hline Osasco & 1,012 & $1,008-1,015$ & 1,012 & $1,007-1,017$ & 1,001 & $0,997-1,004$ \\
\hline São Bernardo & 1,032 & $1,024-1,040$ & 1,033 & $1,019-1,046$ & 1,011 & $1,005-1,018$ \\
\hline São Caetano & 1,015 & $1,006-1,024$ & - & - & 0,994 & $0,985-1,003$ \\
\hline São Paulo & 1,011 & $1,009-1,013$ & 1,017 & $1,015-1,019$ & 1,005 & $1,003-1,006$ \\
\hline Santo André & 0,997 & $0,992-1,002$ & 1,009 & $1,001-1,017$ & 0,991 & $0,987-0,996$ \\
\hline Taboão da Serra & 1,014 & $1,003-1,026$ & 1,015 & $0,997-1,034$ & 1,038 & $1,026-1,050$ \\
\hline
\end{tabular}

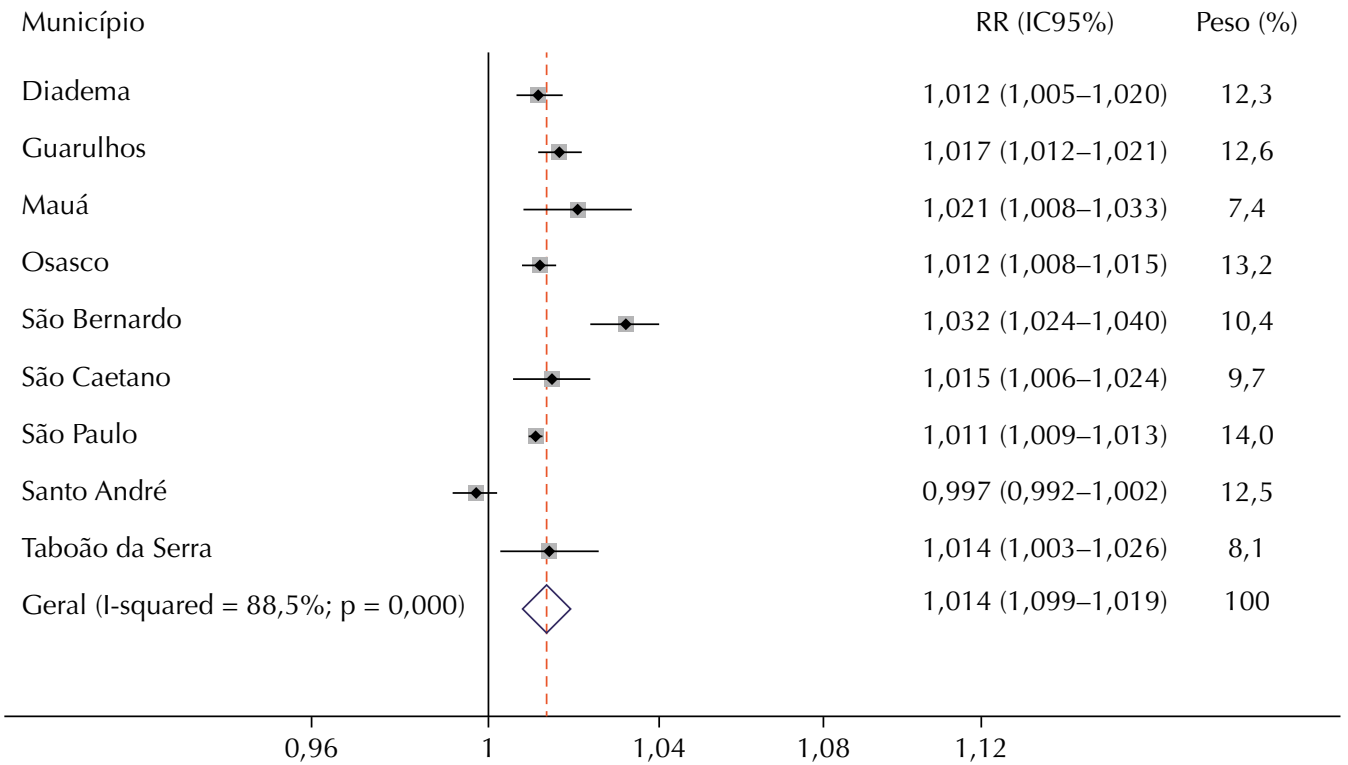

Nota: Pesos da análise de efeitos aleatórios.

Figura 2. Riscos relativos (RR) e intervalo de confiança (IC95\%) de hospitalizações por doenças respiratórias para um aumento de $10 \mu \mathrm{g} / \mathrm{m}^{3}$ nos níveis de $\mathrm{PM}_{10}$ nos municípios estudados e meta-análise para o conjunto de municípios. 


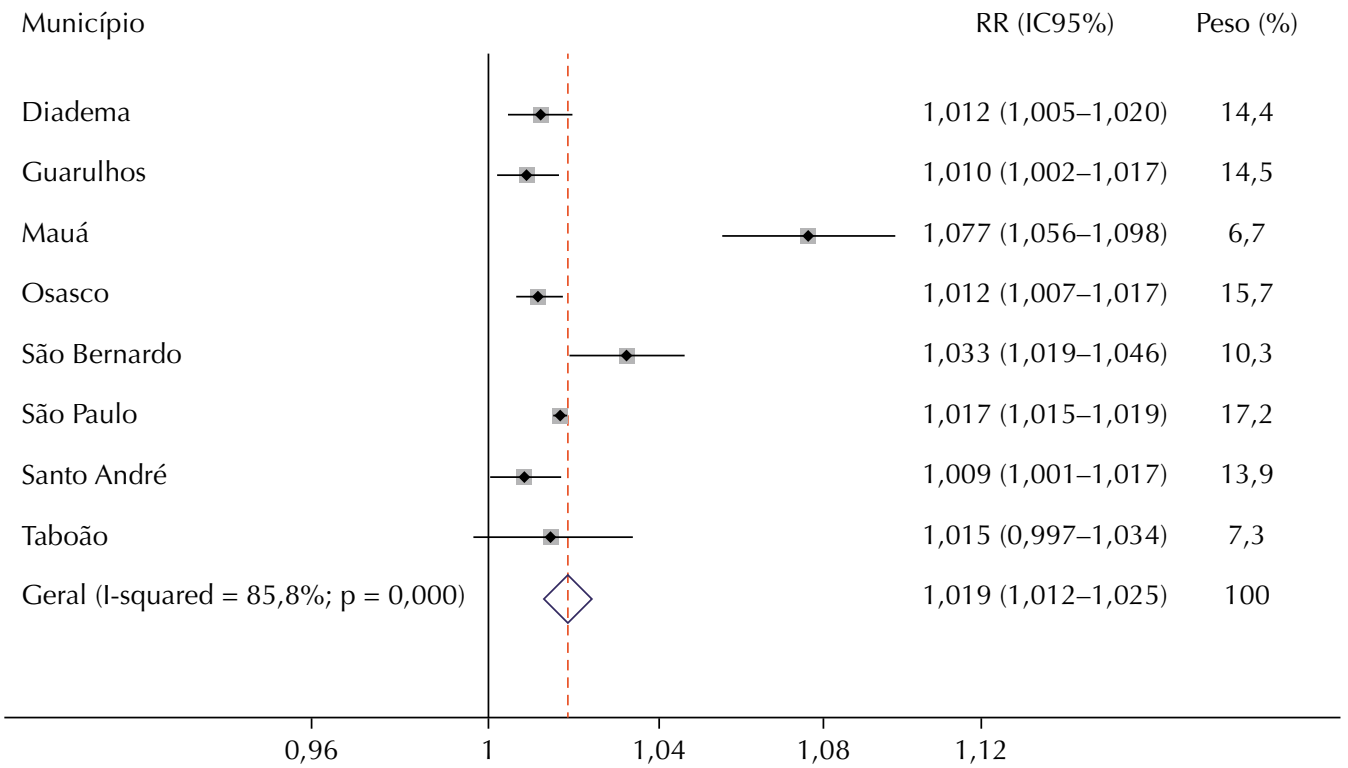

Nota: Pesos da análise de efeitos aleatórios.

Figura 3. Riscos relativos (RR) e intervalo de confiança (IC95\%) de hospitalizações por doenças respiratórias em crianças para um aumento de $10 \mu \mathrm{g} / \mathrm{m}^{3}$ nos níveis de $\mathrm{PM}_{10}$ nos municípios estudados e meta-análise para o conjunto de municípios.

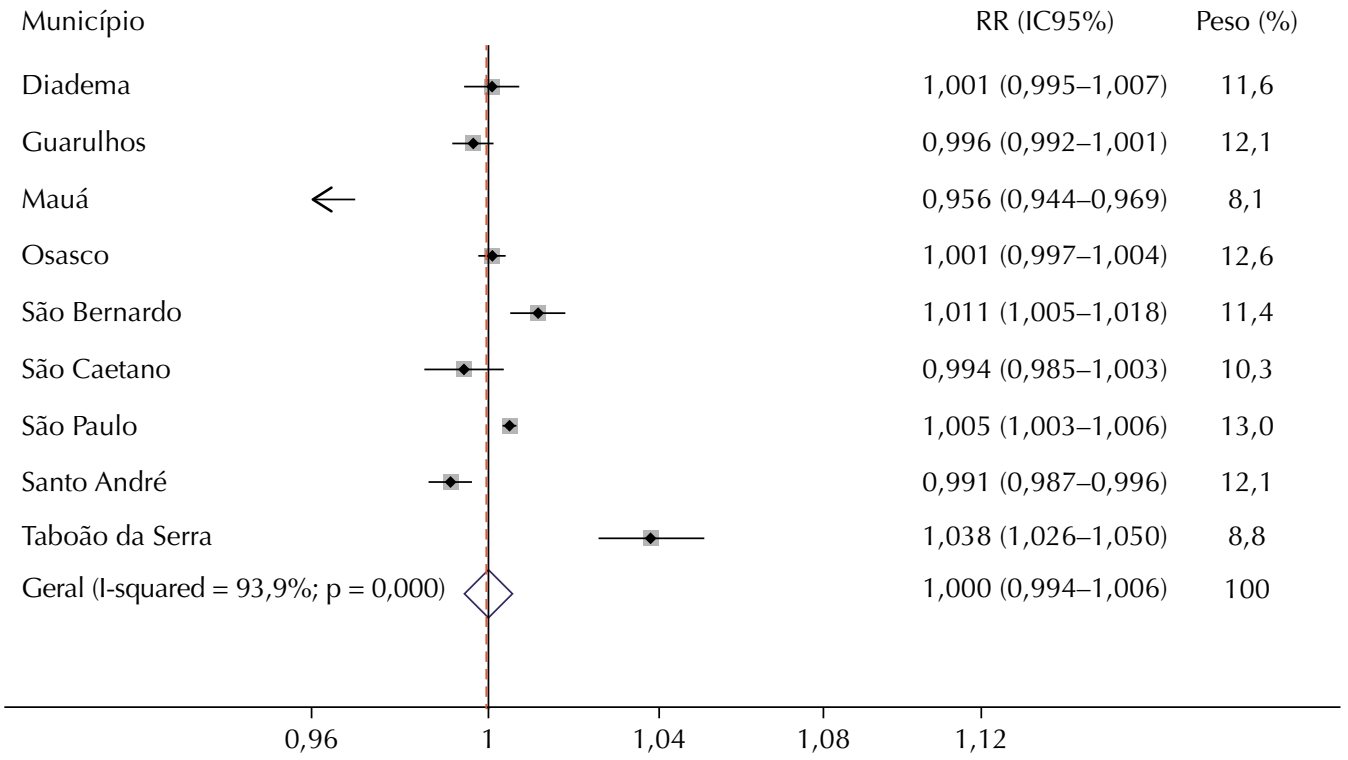

Nota: Pesos da análise de efeitos aleatórios.

Figura 4. Riscos relativos e intervalo de confiança (IC95\%) de hospitalizações por doenças cardiovasculares para um aumento de $10 \mu \mathrm{g} / \mathrm{m}^{3}$ nos níveis de $\mathrm{PM}_{10}$ nos municípios estudados e meta-análise para o conjunto de municípios.

\section{DISCUSSÃO}

Esse é o primeiro estudo a estimar o risco de adoecimento devido à poluição do ar no conjunto de municípios da RMSP. Observamos associações positivas e estatisticamente significantes entre exposição ao $\mathrm{PM}_{10}$ e as internações por doenças respiratórias totais e em crianças menores de cinco anos em quase todos os nove municípios que contam com rede de monitoramento da qualidade do ar nessa região. Por outro lado, apenas os municípios de São Paulo e de São Bernardo do Campo mostraram associação entre os níveis de $\mathrm{PM}_{10}$ e as internações por DCV. De modo similar, as estimativas globais do efeito da exposição à 
poluição na região indicaram associações somente com as doenças respiratórias. Em resumo, observou-se que há um aumento de 1,4\% nas internações por doenças respiratórias totais a cada acréscimo de $10 \mu \mathrm{g} / \mathrm{m}^{3}$ nos níveis de $\mathrm{PM}_{10}$. Para crianças menores de cinco anos, o efeito é ligeiramente maior, havendo um aumento de 1,9\% nas internações.

A magnitude dos efeitos, tanto das estimativas globais quanto daquelas relativas a cada municipío analisado, mostrou-se consistente com outros estudos similares, tanto nacionais ${ }^{8,10}$ quanto realizados em outros países ${ }^{4,11}$. Por exemplo, estudo realizado no município de São Paulo encontrou aumento de 2,4\% nas internações por doenças respiratórias em crianças menores de cinco anos associado a um aumento de $10 \mu \mathrm{g} / \mathrm{m}^{3}$ nos níveis de $\mathrm{PM}_{10}{ }^{10}$. Esse mesmo estudo encontrou associação estatisticamente significante para as internações por DCV e com magnitude muito próxima ao que observamos na presente análise para o município de São Paulo.

Observa-se também que as doenças respiratórias parecem ser o melhor indicador dos efeitos à saúde associados à poluição do ar. Freitas et al. ${ }^{8}$, em estudo conduzido em diversos municípios brasileiros, identificaram as internações por doenças respiratórias em menores de cinco anos, seguidas das internações por doenças respiratórias totais, como os melhores indicadores de efeito à saúde da poluição atmosférica para fins de vigilância.

Nota-se ainda o alto grau de contaminação atmosférica ao qual a população da RMSP está exposta. Dois municípios, Guarulhos e Osasco, apresentaram média anual de $\mathrm{PM}_{10}$ superior ao padrão de qualidade do ar estabelecido pelo Conselho Nacional de Meio Ambiente (CONAMA), equivalente a $50 \mu \mathrm{g} / \mathrm{m}^{3}$. Entretanto, cabe destacar que o Conselho Estadual do Meio Ambiente (CONSEMA) deliberou que o estado de São Paulo deve adotar padrões mais rígidos para a qualidade do $\mathrm{ar}^{22}$. Em maio de 2011, foram adotados novos padrões de qualidade do ar para o estado de São Paulo, com vistas a alcançar os limites recomendados pela Organização Mundial da Saúde, de $20 \mu \mathrm{g} / \mathrm{m}^{3}$, como média anual máxima para o $\mathrm{PM}_{10}$. Ressalta-se que, segundo esse novo parâmetro, ainda não totalmente em vigor no estado, todos os municípios da RMSP estariam em desacordo.

Como qualquer estudo epidemiológico, a presente análise apresenta algumas limitações, como o fato de somente 10 entre 39 municípios da RMSP contarem com monitoramento da qualidade do ar. Além disso, muitos dos municípios apresentam apenas um monitor, que pode não estar refletindo adequadamente a exposição da população ali moradora. Outra limitação é o fato de muitos residentes nesses municípios passarem a maior parte do dia em outras localizações, principalmente nas regiões mais centrais de São Paulo, por motivos de trabalho ou estudo.

Por outro lado, as estimativas obtidas pela meta-análise devem estar refletindo o verdadeiro impacto da poluição atmosférica nos residentes da RMSP. Conhecer e dimensionar as estimativas do impacto global da poluição do ar na população da RMSP é importante para auxiliar o delineamento de intervenções que objetivem diminuir a exposição da população à poluição do ar, assim como estimar os custos econômicos dos efeitos à saúde decorrentes dessa exposição. Essas informações podem, ainda, subsidiar o acompanhamento das variações nos efeitos de saúde decorrentes dessas possíveis intervenções. No momento em que a qualidade do ar ganha cada vez mais destaque como importante determinante da saúde das populações, as cidades devem buscar garantir uma melhor qualidade do ar e, consequentemente, uma melhor qualidade de vida para seus habitantes.

\section{REFERÊNCIAS}

1. Amancio CT, Nascimento LF. Environmental pollution and deaths due to stroke in a city with low levels of air pollution: ecological time series study. Sao Paulo Med J. 2014;132(6):353-8. https://doi.org/10.1590/1516-3180.2014.1326733 
2. Baccarelli A, Martinelli I, Zanobetti A, Grillo P, Hou LF, Bertazzi PA, et al. Exposure to particulate air pollution and risk of deep vein thrombosis. Arch Intern Med. 2008;168(9):920-7. https://doi.org/10.1001/archinte.168.9.920

3. Barbosa SMM, Farhat SCL, Martins LC, Pereira LAA, Saldiva PHN, Zanobetti A, et al. Air pollution and children's health: sickle cell disease. Cad Saude Publica. 2015;31(2):265-75. https://doi.org/10.1590/0102-311X00013214

4. Carugno M, Consonni D, Randi G, Catelan D, Grisotto L, Bertazzi PA, et al. Air pollution exposure, cause-specific deaths and hospitalizations in a highly polluted Italian region. Environ Res. 2016;147:415-24. https://doi.org/10.1016/j.envres.2016.03.003

5. Chiarelli PS, Pereira LAA, Saldiva PHN, Ferreira Filho C, Garcia MLB, Braga ALF, et al. The association between air pollution and blood pressure in traffic controllers in Santo André, São Paulo, Brazil. Environ Res. 2011;111(5):650-5. https://doi.org/10.1016/j.envres.2011.04.007

6. DerSimonian R, Laird N. Meta-analysis in clinical trials. Control Clin Trials. 1986;7(3):177-88. https://doi.org/10.1016/0197-2456(86)90046-2

7. Evo CPR, Ulrych BK, Takegawa B, Soares G, Nogueira G, Oliveira LO, et al. Poluição do ar e internação por insuficiência cardíaca congestiva em idosos no município de Santo André. Arq Bras Cienc Saude. 2011 [citado 10 jul 2017];36(1):6-9. Disponível em: http://files.bvs.br/ upload/S/1983-2451/2011/v36n1/a1917.pdf

8. Freitas CU, Junger W, Ponce de Leon AC, Grimaldi R, Silva MAFR, Gouveia N. Poluição do ar em cidades brasileiras: selecionando indicadores de impacto na saúde para fins de vigilância. Epidemiol Serv Saude. 2013;22(3):445-54. https://doi.org/10.5123/S1679-49742013000300009

9. Gouveia N, Bremner SA, Novaes HMD. Association between ambient air pollution and birth weight in São Paulo, Brazil. J Epidemiol Community Health. 2004;58(1):11-7. https://doi.org/10.1136/jech.58.1.11

10. Gouveia N, Freitas CU, Martins LC, Marcilio IO. Hospitalizações por causas respiratórias e cardiovasculares associadas à contaminação atmosférica no Município de São Paulo, Brasil. Cad Saude Publica. 2006;22(12):2669-77. https://doi.org/10.1590/S0102-311X2006001200016

11. Guo Y, Tong S, Zhang Y, Barnett AG, Jia Y, Pan X. The relationship between particulate air pollution and emergency hospital visits for hypertension in Beijing, China. Sci Total Environ. 2010;408(20):4446-50. https://doi.org/10.1016/j.scitotenv.2010.06.042

12. Lima TAC, Nascimento LFC, Medeiros APP, Santos VP. Association between maternal exposure to particulate matter and premature birth. Rev Ambient Agua. 2014;9(1):27-36. https://doi.org/10.4136/ambi-agua.1262

13. Lin CA, Pereira LAA, Nishioka DC, Conceição GMS, Braga ALF, Saldiva PHN. Air pollution and neonatal deaths in São Paulo, Brazil. Braz J Med Biol Res. 2004;37(5):765-70. https://doi.org/10.1590/S0100-879X2004000500019

14. Medeiros A, Gouveia N. Relação entre baixo peso ao nascer e a poluição do ar no Município de São Paulo. Rev Saude Publica. 2005;39(6):965-72. https://doi.org/10.1590/S0034-89102005000600015

15. Nascimento LFC, Francisco JB, Patto MBR, Antunes AM. Environmental pollutants and stroke-related hospital admissions. Cad Saude Publica. 2012;28(7):1319-24. https://doi.org/10.1590/S0102-311X2012000700010

16. Negrete BR, Rosa CC, Ikeuti DH, Delena PJ, Borba TM, Braga ALF. Poluição atmosférica e internações por insuficiência cardíaca congestiva em adultos e idosos em Santo André (SP). Arq Bras Cienc Saude. 2010 [citado 10 jul 2017];35(3):208-12. Disponível em: http://files.bvs.br/ upload/S/1983-2451/2010/v35n3/a1688

17. Nicolussi FH, Santos APM, André SCS, Veiga TB, Takayanagui AMM. Poluição do ar e doenças respiratórias alérgicas em escolares. Rev Saude Publica. 2014;48(2):326-30. http://doi.org/10.1590/S0034-8910.2014048004940

18. Poursafa P, Kelishadi R, Lahijanzadeh A, Modaresi M, Javanmard SH, Assari R, et al. The relationship of air pollution and surrogate markers of endothelial dysfunction in a population-based sample of children. BMC Public Health. 2011;11:115. https://doi.org/ 10.1186/1471-2458-11-115

19. Romão R, Pereira LAA, Saldiva PHN, Pinheiro PM, Braga ALF, Martins LC. The relationship between low birth weight and exposure to inhalable particulate matter. Cad Saude Publica. 2013;29(6):1101-8. https://doi.org/10.1590/S0102-311X2013000600007

20. Santos UP, Terra-Filho M, Lin CA, Pereira LA, Vieira TC, Saldiva PH, et al. Cardiac arrhythmia emergency room visits and environmental air pollution in Sao Paulo, Brazil. J Epidemiol Community Health. 2008;62(3):267-72. https://doi.org/10.1136/jech.2006.058123 
21. Schwartz J. The distributed lag between air pollution and daily deaths. Epidemiology. 2000;11(3):320-6. https://doi.org/10.1097/00001648-200005000-00016

22. Valentim LSO. Consema aprova novos padrões de qualidade do ar para São Paulo. BEPA Bol Epidemiol Paulista. 2011 [citado 10 jul 2017];8(91):27-9. Disponível em: http://www.cvs. saude.sp.gov.br/up/Consema\%20aprova $\% 20$ novos $\% 20 \% 20 \% 20$ padr\%C3\%B5es\%20de \%20 qualidade\%20do\%20ar\%20para\%20S\%C3\%A30\%20Paulo.pdf

23. Yanagi Y, Assunção JV, Barrozo LV. The impact of atmospheric particulate matter on cancer incidence and mortality in the city of São Paulo, Brazil. Cad Saude Publica. 2012;28(9):1737-48. https://doi.org/10.1590/S0102-311X2012000900012

Financiamento: Ministério da Saúde (MS - Processo 1297/2008)

Contribuição dos Autores: Concepção e planejamento do estudo, análise e interpretação dos dados: NG, ACPL, WJ, CUF. Coleta de dados: FPC, CUF. Todos os autores contribuíram na elaboração e revisão do manuscrito, aprovaram sua versão final e assumem responsabilidade pública pelo seu conteúdo.

Conflito de Interesses: Os autores declaram não haver conflito de interesses. 